\title{
Die maßlose Darstellung von Bildern
}

\author{
ARNO SCHUBBACH
}

Inwieweit Bilder maßlos sein können, hängt von der Bestimmung des Bildlichen ab. Bilder begreifen wir meist als das, was uns sichtbar vor Augen steht, so dass ihre Maßlosigkeit im Dargestellten, in eingesetzten Mitteln oder im Spiel mit Konventionen zu suchen wäre. Da viele und vor allem epistemische Bilder etwas darzustellen beanspruchen, ist es jedoch erforderlich, die Verfahren der Bildherstellung einzubeziehen. Das bildliche Bedeuten muss nicht nur ausgehend von der Betrachtung, sondern auch in deren Bezug zur Herstellung des Bildes gefasst werden. An André Skupins Karte der "World of Geography" soll exemplarisch gezeigt werden, wie die Verfahren der Visualisierung mit der Form der Darstellung sichtbar in Widerstreit geraten können. Maßlosigkeit ist daher nicht allein im Sichtbaren, sondern ebenso in dessen Verhältnis zur Sichtbarmachung aufzusuchen. Sie hat ibren Ort zwischen der Darstellung von Bildern als solchen und der Darstellung von etwas durch Bilder.

Neuere Arbeiten zur Ästhetik und das Vorhaben der Bildwissenschaft oder Bildkritik haben durch die Ausweitung ihrer Grundbegriffe von sich reden gemacht: Die Ästhetik löst sich zunehmend von ihrer engen Fassung als philosophische Lehre von der Kunst und dem Schönen, um sich neue Gebiete wie die Wissenschaften zu erschließen ${ }^{1}$ oder affektiv getönte Wahrnehmungen, ${ }^{2}$ besondere Aufmerksamkeit erheischende Erscheinungen ${ }^{3}$ oder ein Denken im engen Bezug zur sinnlichen Erfahrung zu konzipieren. ${ }^{4}$ Der Begriff des Bildes ist dagegen in erster Linie seitens der Kunstgeschichte als zentrale interdisziplinäre Konzeption vorgeschlagen worden, um das an Kunstwerken entfaltete Wissen in einen größeren Zusammenhang zu stellen und eine allgemeinere Erforschung von Bildern zum

1 Siehe exemplarisch Wolfgang Krohn (Hg.): Ästhetik in der Wissenschaft. Interdisziplinärer Diskurs über das Gestalten und Darstellen von Wissen, Hamburg 2006.

2 Siehe Gernot Böhme: Aisthetik. Vorlesungen über Ästhetik als allgemeine Wahrnehmungslehre, München 2001, insbesondere S. 73.

3 Siehe Martin Seel: Ästhetik des Erscheinens, Frankfurt am Main 2003, insbesondere S. 49-69.

4 Siehe Wolfgang Welsch: Ästhetisches Denken, Stuttgart 1990, S. 41-78. 
Programm zu machen. 5 Das `Ästhetische` und das `Bild ‘ scheinen somit an Aktualität zu gewinnen, insofern sie nicht auf die Kunst beschränkt werden und die an der Kunst geschulten Instrumentarien in einem erweiterten Umkreis fruchtbar machen können.

In der Folge wird zu klären sein, wie die Begriffe des Ästhetischen und des Bildes neu zu fassen und ins Verhältnis zu setzen sind. Diese Frage wird anhand konkreter Beispiele zu verhandeln sein, weil Ästhetik und Bildkritik auf den wesentlichen Bezug des Denkens zur sinnlichen Konkretion setzen. Zugleich verbietet sich eine Verengung der Frage auf künstlerische Bilder, weil die Begriffe des Ästhetischen und des Bildes ihre Aktualität in erster Linie mit Hinblick auf Gegenstände und Themen jenseits der Kunst gewinnen. Die Herausforderung, diese Aktualität auch tatsächlich unter Beweis zu stellen, ist aber umso größer, je weiter man sich von der Kunst entfernt. Dennoch ist sie beispielsweise mit Blick auf wissenschaftliche Bilder angenommen worden und hat zu ebenso vielfältigen wie produktiven Beiträgen zum Verständnis der Wissenschaften Anlass gegeben. Die Rolle von Bildern für die heutige Forschungspraxis und in den angewandten Wissenschaften wurde ebenso herausgearbeitet wie die ästhetische Dimension von Visualisierungen. ${ }^{6}$ Als produktiv haben sich dabei vor allem Untersuchungen erwiesen, die die Begriffe der Ästhetik und des Bildes nicht einfach vorausgesetzt und auf die Wissenschaften angewendet, sondern zugleich kritisch hinterfragt und konzeptionellen Revisionen unterworfen haben.

Die Frage nach der Maßlosigkeit von Bildern und einer Ästhetik der Transgression kann vor diesem Hintergrund als eine besondere Herausforderung gesehen werden. Denn es liegt zunächst nah, bei diesen Formulierungen an künstlerische Bilder zu denken, die vielleicht etwas Schockierendes zeigen oder Drastisches

5 Siehe stellvertretend Gottfried Boehm (Hg.): Was ist ein Bild?, München 1994. Hans Belting: Bild-Anthropologie. Entwürfe für eine Bildwissenschaft, München 2001. Horst Bredekamp: Artikel >Bildwissenschaft. In: Ulrich Pfisterer (Hg.): Metzler Lexikon Kunstwissenschaft. Ideen, Methoden, Begriffe, Stuttgart, Weimar 2003, S. 56-58. James Elkins: The Domain of Images, Ithaca, London 1999.

6 Es wäre hier eine Vielzahl von Arbeiten zu nennen. Ich möchte aber wiederum nur exemplarisch verweisen auf die einschlägigen Aufsätze in Bettina Heintz, Jörg Huber (Hg.): Mit dem Auge denken. Strategien der Sichtbarmachung in wissenschaftlichen und virtuellen Welten, Zürich 2001. Peter Geimer (Hg.): Ordnungen der Sichtbarkeit. Fotografie in Wissenschaft, Kunst und Technologie, Frankfurt am Main 2002 sowie in Michael Lynch, Steve Woolgar (Hg.): Representation in Scientific Practice, Cambridge et al. 1990. Siehe darüber hinaus Michael Lynch, Samuel Y. Edgerton Jr.: Aesthetics and Digital Image Processing: Representational Craft in Contemporary Astronomy. In: Gordon Fyfe, John Law (Hg.): Picturing Power: Visual Depiction and Social Relations, London, New York 1988, S. 184-220 und Michael Lynch: Discipline and the Material Form of Images: An Analysis of Scientific Visibility. In: Social Studies of Science 15 (1985), S. 37-66 sowie Lorraine Daston, Peter Galison: Objectivity, New York 2007. Peter Galison: Image \& Logic. A Material Culture of Microphysics, Chicago, London 1997. 
darstellen, die anstößige Materialien einsetzen und Konventionen angreifen, sich ihrer Deutung in einer reflexiven Wendung oder in einem aufdringlichen Hyperrealismus verweigern. Die meisten Leser mögen auch an eine `Ästhetikı der Gewalt von besonders grausamen oder gar gewalttätigen Filmen oder der seit einigen Jahren populär diskutierten Killerspieler denken. Im Hinblick auf die Diskussionen um die erweiterten Begriffe des Bildes und des Ästhetischen verengen solche Assoziationen den Blick auf das konzeptionelle wie heuristische Potential der beiden Begriffe. Es lohnt sich daher, einmal von den Rändern aus zu fragen: Können beispielsweise wissenschaftliche Bilder und ihre Ästhetik als maßlos begriffen werden? Diese Frage möchte ich im Folgenden erörtern, um sie schärfer zu konturieren.

\section{Maßlose Projektionen zwischen Kugelfläche und Kartenblatt}

Betrachten wir als einleitendes Beispiel zwei Weltkarten (Abb.1 und Abb. 2). Auf den ersten Blick dürften sie wohl kaum jemandem als maßlos erscheinen, zumal Karten auch dem praktischen Zweck dienen, die Welt, von der wir umgeben sind und in der wir uns orientieren müssen, überschauen zu können. Ein Vergleich der beiden Karten wirft jedoch durchaus Fragen auf. Eine Karte wirkt auf uns vertraut, die zweite aber scheint vor allem an den Polen auf irritierende Weise länglich gestreckt. Dieser Unterschied begründet sich in den voneinander abweichenden Herstellungsweisen: In der Kartografie spielte seit langem die Mercator-Projektion eine zentrale Rolle, weil sie aufgrund ihrer Winkeltreue vor allem für die Navigation praktische Vorteile hat (Abb. 1). ${ }^{7}$ Der Preis für diesen Vorzug sind enorme Verzerrungen der Flächenverhältnisse, da alle Flächen um den Äquator verkleinert und zu den Polen hin vergrößert werden. Dieser Umstand erregte aufgrund der praktischen Bedeutung der Mercator-Karte und ihrer weiten Verbreitung immer wieder Kritik, ${ }^{8}$ auch wenn bereits seit Mercators Zeiten ebenso Projektionen bekannt waren, die die Flächenverhältnisse erhalten. ${ }^{9}$ In den 1960er Jahren wurde diese Diskussion durch Arno Peters schließlich in die breitere Öffentlichkeit getragen, als er mit politischer Verve die fälschliche Verkleinerung der DritteWelt-Länder angriff und dagegen eine flächentreue Projektion vorschlug, die stattdessen die Winkeltreue aufgibt (Abb. 2). ${ }^{10}$ Die vorgebrachte Projektion war allerdings seit dem 19. Jahrhundert bekannt. ${ }^{11}$

7 Siehe John P. Snyder: Flattening the Earth. Two Thousand Years of Map Projections, Chicago, London 1993, S. 43-49.

8 Siehe ebd., S. 156-157.

9 Siehe zur Sinusoidal-Projektion ebd., S. 49-51.

10 Siehe Arno Peters: Die neue Kartographie - The new Cartography, Klagenfurt, New York 1983, S. 50-129.

11 Siehe zu Arno Peters' Intervention und James Galls Projektion Snyder 1993 (wie Anm. 7), S. 164-166. 


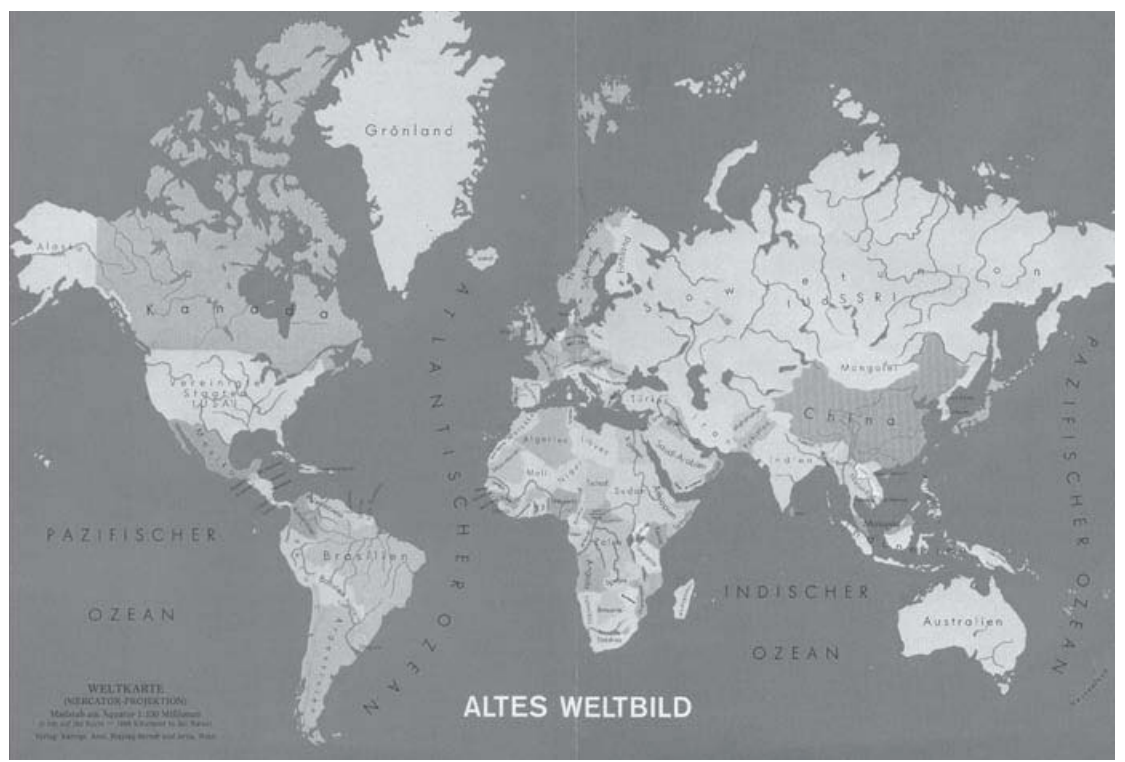

Abb. 1: Weltkarte nach Gerhard Mercator (1512-1594) aus Arno Peters: Die perspektivische Verzerrung von Raum und Zeit im historisch-geographischen Weltbilde der Gegenwart und ihre Überwindung durch neue Darstellungsweisen, München-Solln 1967.

Diese Beschreibung geht von der Herstellung der Karten aus und stellt mit dem Begriff der Projektion die Abbildung ins Zentrum, die geografische Größenverhältnisse in sichtbare Relationen im Bild transformieren soll. Diese Abbildung ist indes allzu ungesichert, weil im Bild realer Verhältnisse verloren gehen und zugleich neue, in diesem Fall täuschende grafische Verhältnisse entstehen. Diese Beobachtung beschreibt ein prinzipielles Problem von geografischen Karten, wie bereits Leonhard Euler gezeigt hat: Keine Projektion der gekrümmten Erdoberfläche auf das ebene Kartenbild erlaubt es, gleichzeitig Längen, Flächen und Winkel zu erhalten. Da die berechnete Karte aber notwendigerweise Längen, Flächen und Winkel darstellen muss, wird sie zumindest in einer Hinsicht in die Irre führen. ${ }^{12}$ Der Übergang von den Größenverhältnissen in der Welt zu den Proportionen auf der Bildfläche erfordert daher kartografische Entscheidungen und impliziert im entstehenden Bild die Möglichkeit täuschender Effekte: Damit bestimmte grafische Beziehungen realer Verhältnisse in der Welt darstellen können, muss in Kauf genommen werden, dass andere Beziehungen im Bild nicht

12 Siehe ebd., S. 73-74 und Leonhard Euler: Drei Abhandlungen über Kartenprojection, hg. von A. Wangerin, Leipzig 1898, S. 8-10. 


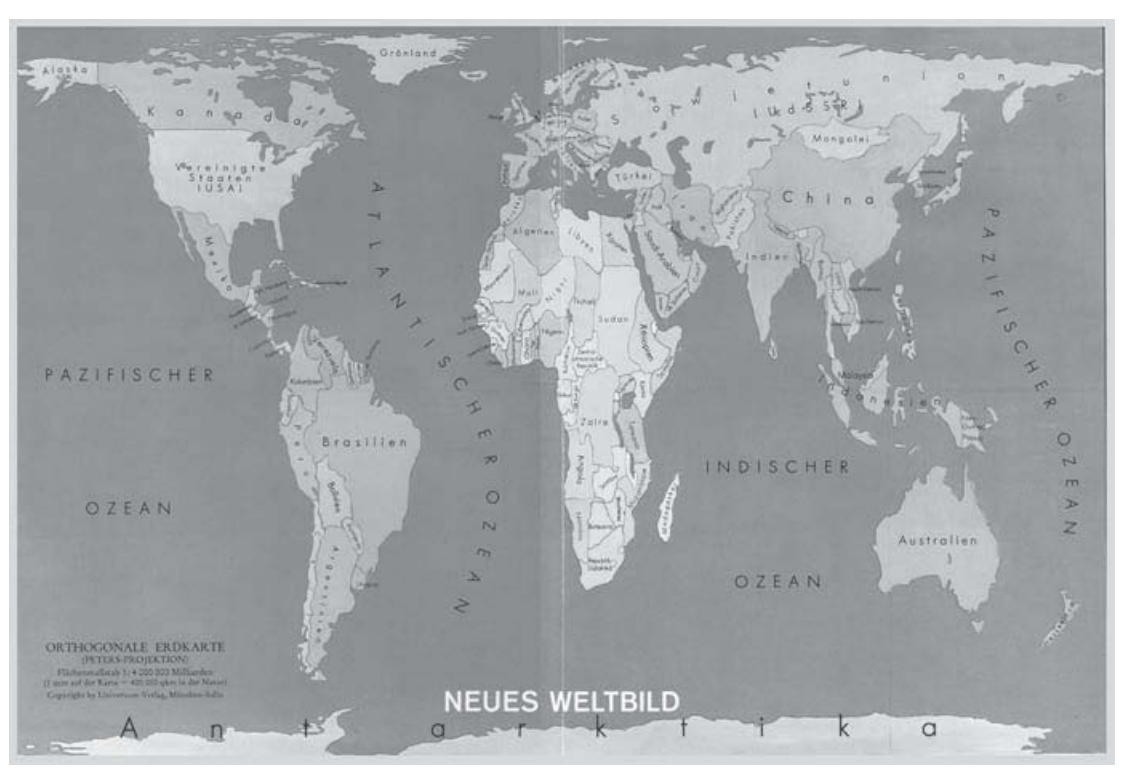

Abb.2: Weltkarte nach Arno Peters: Die perspektivische Verzerrung von Raum und Zeit historischgeographischen Weltbilde der Gegenwart und ihre Überwindung durch neue Darstellungsweisen, München-Solln 1967.

zutreffend darstellen. Durch Herstellungsverfahren wie die Projektion wird zwar versucht, darstellende und dargestellte Bezüge eins zu eins aufeinander abzubilden, im Allgemeinen kann aber ebenso wenig wie in der Kartografie vorausgesetzt werden, dass dies überhaupt möglich ist.

Infolgedessen lässt sich die Vermutung wagen, dass Bilder nicht nur wegen des Dargestellten oder der eingesetzten Mittel maßlos genannt werden können. An den beiden Weltkarten zeigt sich nämlich, dass die Maßlosigkeit ihren Ort nicht ausschließlich im Sichtbaren, sondern auch im Bezug des Bildes auf seine Herstellung haben kann. Diese Karten wären demnach insofern maßlos zu nennen, als das sichtbare Bild in seinen grafischen Konstellationen etwas darstellt, ohne dass durch das Bild selbst gegeben wäre, welche grafischen Relationen tatsächlich etwas darstellen und welche nicht. An den vorangehenden Beispielen wird dies deutlicher: Die Mercator-Projektion ist nicht flächentreu, muss aber doch Größenverhältnisse der Kontinente, Meere und Nationalstaaten zeigen; im Falle der Peters-Projektion gilt ähnliches für die Winkel auf der Kugeloberfläche und im Kartenbild. Beide Projektionen sind daher insofern maßlos, als die Karte unter anderem auch Beziehungen darstellt, denen keine tatsächlichen Größenverhältnisse im dargestellten Raum entsprechen. Manches in den Daten kann nicht ins Bild gesetzt werden, zugleich werden im Bild grafische Beziehungen geschaffen, die dann etwas 
darzustellen scheinen, was aufgrund der vorhandenen Daten anders zu bestimmen wäre oder unbestimmt bleiben müsste. ${ }^{13}$

In dieser Hinsicht von maßlosen Bildern zu reden, impliziert natürlich keine prinzipielle, vollkommene Maßlosigkeit eines Bildes oder gar von Bildern im Allgemeinen. Vielmehr akzentuiert diese Beschreibung das spezifische Problem von Darstellungen, einen Bezug zwischen dem vor Augen stehenden, sichtbaren Bild und dem im Bild sichtbar gemachten Gegenstand zu eröffnen, diesen Bezug aber nicht selbst transparent machen zu können. Zwar zielen die methodischen Diskussionen in den Wissenschaften stets darauf ab, die Verfahren der Bildgebung und den Übergang von den diversen Daten zum Bild zu standardisieren und dadurch zu kontrollieren, was im Bild als belastbare Information gelten kann. Jedoch steht in Frage, ob dies zu gewährleisten ist, wo Erkenntnisse auf visuellem Wege erlangt werden sollen und vom Bild ihren Ausgang nehmen. Zudem werden wir uns dem visuellen Eindruck und seinen Evidenzen kaum vollständig entziehen können, auch wenn wir wissen mögen, dass der Darstellung in mancher Hinsicht nicht zu trauen ist. ${ }^{14}$

Diese systematischen Probleme wurden in erster Linie von Studien zu wissenschaftlichen Visualisierungen herausgearbeitet. Sie haben die Verfahren zur Sichtbarmachung in den Vordergrund gerückt und in die Diskussion des Bildes eingeführt, weil diese Verfahren für die Gültigkeit von visuell gewonnener Erkenntnis entscheidend sind. ${ }^{15}$ Damit tritt aber ein Aspekt hervor, der von allgemeinerer Bedeutung ist: Die Herstellung ist kein vernachlässigbarer Faktor für die Betrachtung eines Bildes. Bilder müssen nicht zeigen, wie sie gemacht wurden, dennoch

13 Es ist dabei nicht entscheidend, ob das Bild ein Abbild in dem Sinne zu sein scheint, dass das Dargestellte unabhängig und vorgängig zu seiner Darstellung existiert. Das Dargestellte mag in der Darstellung entstehen oder vielleicht auch fingiert sein - solange sich in den grafischen Konfigurationen des Bildes ein inhärentes Gefüge dessen zeigen soll, was das Bild darstellt, stellt sich die Frage nach dem Verhältnis von darstellenden und dargestellten Zügen.

14 Jochen Hennig zeigt an der Entwicklung der Rastertunnelmikroskopie in den 1980er Jahren beispielhaft, wie die Erwartung, Atome müssten sich über die Oberfläche erheben, zur Etablierung der Konvention beitrug, sie als Erhebungen darzustellen, obwohl die Messwerte sie als elektrische Maxima oder Minima ausweisen. Siehe Jochen Hennig: Changes in the Design of Scanning Tunneling Microscopic Images from 1980 to 1990. In: Techné 8 (2004), S. 3655; hier S. 44-48. Die Darstellung wurde damit als ein vermeintlich realistisches Bild etabliert und evoziert wiederum die entsprechenden Erwartungen des Blicks, auch wenn den Physikern der Messprozess und seine Gesetzlichkeiten bekannt sein mögen.

15 Der Begriff der Sichtbarmachung wurde bekanntermaßen von Hans-Jörg Rheinberger geprägt. Siehe zum Beispiel Hans-Jörg Rheinberger: Objekt und Repräsentation. In: Bettina Heintz, Jörg Huber (Hg.): Mit dem Auge denken. Strategien der Sichtbarmachung in wissenschaftlichen und virtuellen Welten, Zürich 2001, S. 55-61 und ders.: Spurenlesen im Experimentalsystem. In: Sybille Krämer, Werner Kogge, Gernot Grube (Hg.): Spur. Spurenlesen als Orientierungstechnik und Wissenskunst, Frankfurt am Main 2007, S. 293-308; insbesondere S. 298-307. 
betrachten wir sie als gemachte. Ihr Bedeuten ist niemals unabhängig von der Dimension ihrer Herstellung und des Anscheins der eingesetzten Verfahren und Mittel im Bild. Dieser Ansatz umreißt eine Topologie des Bildlichen, in der Bilder zugleich als hergestellte Artefakte und als betrachtete Darstellungen zu begreifen sind und deshalb eine Behandlung erfordern, die sie weder auf die Herstellung von Artefakten noch auf die Betrachtung des bloß sichtbaren Resultats reduziert. ${ }^{16}$

Die Herstellung spielt in der Frage des Bildes allerdings eine doppelte Rolle. Zum einen kann sie wie bei jedem beliebigen Artefakt als bloße Herstellung thematisiert werden. Sie ist in diesem Fall zwar auf das Bild und seine Darstellung ausgerichtet, charakterisiert aber nicht auf spezifischere Weise seine Betrachtung. Zum anderen kann untersucht werden, wie die Herstellung in die Wahrnehmung des Bildes und in den bildlichen Sinn hineinspielt. Ausgehend von der Betrachtung ist einerseits entscheidend, wie ein Bild tatsächlich entstanden ist und wie sich dieser Prozess sichtbar in das Bild eingeschrieben hat. Andererseits kann die Herstellung auch insofern eine Rolle spielen, als uns das Bild in seiner grafischen Gestaltung eine spezifische Herstellung nahe legt, damit wir es als eine bestimmte Darstellung betrachten und unser visueller Bezug auf den dargestellten Gegenstand eine konkrete Form annimmt. Viele Bilder stellen etwas dar, indem sie uns glauben lassen, dass die grafischen Konfigurationen und Konstellationen auf bestimmte Weise entstanden sind und sie uns deshalb etwas in einer spezifischen Hinsicht zeigen. Insbesondere die Kartendarstellung kann so genutzt werden, um alle möglichen Daten ins Bild zu setzen, was sich in den vielfältigen Formen von thematischen Karten bereits andeutet, in denen eine geografische Karte zusätzlich Daten zu bestimmten Themen visualisiert. Kartendarstellungen können aber auch ganz unabhängig von geografischen Karten erstellt werden. Wenn eine Darstellung aber tatsächlich auf andere Weise entsteht, als sie in ihrer grafischen Gestalt glauben macht, dann kann sie mit ihrer Herstellung sichtbar in Konflikt geraten.

Es zeichnet sich in solch kritischen Momenten im Bild eine Maßlosigkeit ab, die nicht allein im ungesicherten Verhältnis der darstellenden Relationen im Bild und der durch das Bild dargestellten Relationen gründet. Sie hat ihren Ort vielmehr im sichtbaren Rückbezug auf die Herstellung des Bildes, die bei bildgebenden Verfahren den Gegenstandsbezug absichern soll, in Konflikten mit der Form der Darstellung aber auch sichtbar fragwürdig werden kann. An einer abstrakten Karte von Texten möchte ich daher zu zeigen versuchen, dass im Bezug der Herstellungsweisen zum sichtbaren Bild eine eigene Quelle der Maßlosigkeit ausgemacht werden kann. Aus diesem Befund wären nicht nur bildtheoretische Konsequenzen zu ziehen, sondern auch Anforderungen für eine Ästhetik zu folgern, die über den Bereich der Kunst hinausgreift, aber am Phänomen des Bildlichen

16 Siehe dazu ausführlicher Arno Schubbach: Gezogene Linien sehen. Sichtbarmachung und Sichtbarkeit von Bildern. In: Zeitschrift für Ästhetik und Allgemeine Kunstwissenschaft 53 (2008), S. 217-230. 
festhält: Ein Bild ist kein Gegenstand wie jeder andere und ist daher durch eine allgemeine Theorie des Ästhetischen nicht bereits hinreichend behandelt. Vielmehr bringt das Bild eigene Bedingungen mit sich, die in die Bildwahrnehmung eingehen und insbesondere die Dimension der Herstellung beziehungsweise der Sichtbarmachung einschließen. Jede Ästhetik, die den Bereich des Ästhetischen ausweitet, im künstlerischen Bild aber zumindest noch einen paradigmatischen Fall sieht, kann nicht darauf verzichten, sich der unterschiedlichen Spezifik ihrer Gegenstände zu versichern.

\section{Eine maßlose Karte der Geografie}

Die Visualisierung "The World of Geography“ (siehe Abb. 3) erinnert in ihrer grafischen Gestaltung an eine Karte, stellt aber keine reale Geografie dar. ${ }^{17}$ Sie basiert vielmehr auf der Auswertung von allen 2.220 verfügbaren Abstracts der Vorträge, die anlässlich des jährlichen Treffens der Association of American Geographersim Jahr 1999 gehalten wurden. André Skupin, selbst ein ausgebildeter Geograf, hat diese Visualisierung als ein Ergebnis seiner laufenden Forschungen präsentiert und seine Vorgehensweise in einer Reihe von Artikeln ausgeführt. ${ }^{18}$ Es geht ihm um zweierlei. Skupin will uns mit dieser Karte einen "snapshot of the geographic discipline, from established, well-publicized research fields to those only recently emerging ${ }^{19}$ vor Augen halten. Zugleich versucht er damit, den Nutzen von kartografischen Methoden zur Visualisierung von Informationen im Allgemeinen unter Beweis zu stellen, womit er seine Arbeit im Zusammenhang der Informatik und deren Forschungen zur so genannten knowledge domain visualization und information visualization situiert. ${ }^{20}$ Die Geografie spielt in Skupins Arbeit folglich eine doppelte

17 An dieser Stelle möchte ich André Skupin für die Erlaubnis zum Abdruck der Visualisierung danken sowie für seine wertvollen inhaltlichen Hinweise.

18 Siehe André Skupin: A Cartographic Approach to Visualizing Conference Abstracts. In: IEEE Computer Graphics and Applications 22 (2002), S. 50-58. Ders.: The World of Geography. Visualizing a Knowledge Domain with Cartographic Means. In: Proceedings of the National Academy of Sciences 101 (2004), Supplement 1, S. 5274-5278 und ders.: A Picture from a Thousand Words. In: Computing in Science and Engineering 6 (2004), S. 84-88. Diese Artikel sind neben anderen auf der Homepage des Autors verfügbar, http://geography.sdsu. edu/People/Pages/skupin/index.htm (Letzter Zugriff: 10. Oktober 2008).

19 Skupin 2004 (wie Anm. 18), S. 5274.

20 Siehe für einen Überblick die drei Standardwerke Chaomei Chen: Information Visualization. Beyond the Horizon, London et al. ${ }^{2} 2004$. Robert Spence: Information Visualization. Design for Interaction, Harlow et al. ${ }^{2} 2007$. Colin Ware: Information Visualization. Perception for Design, Amsterdam et al. 2004. Siehe für das engere Feld der knowledge domain visualization vor allem Katy Börner, Chaomei Chen, Kevin W. Boyack: Visualizing Knowledge Domains. In: Annual Review of Information Science and Technology 37 (2003), S. 179-255. 


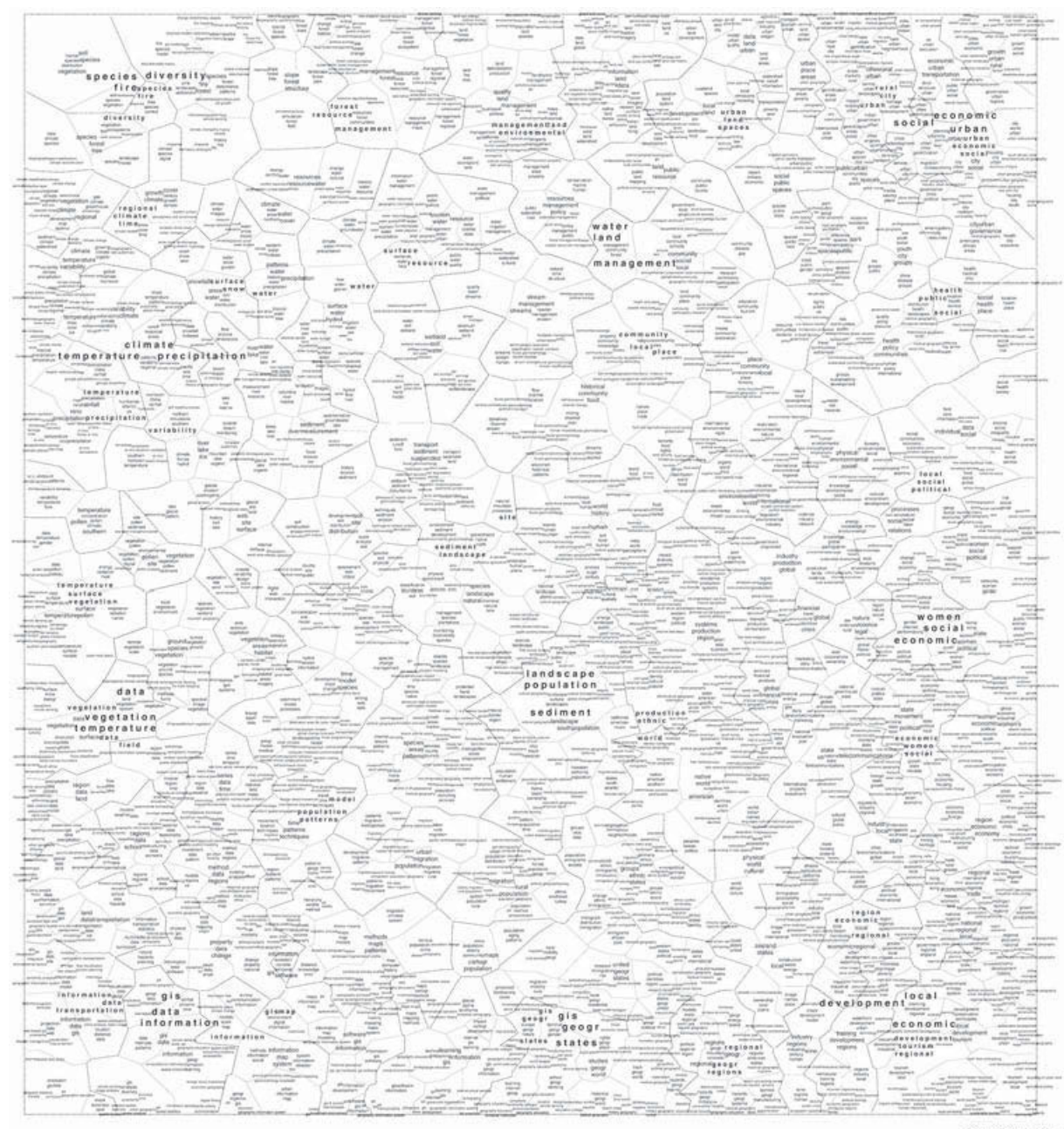

Abb. 3: Visualisierung von 2220 Abstracts der Vorträge am jährlichen Treffen der `Association of American Geographers im Jahr 1999, vorgestellt in André Skupin: The World of Geography: Visualizing a Knowledge Domain with Cartographic Means. In: Proceedings of the National Academy of Sciences 101 (2004), Supplement 1, S. 5274-5278. Siehe auch Farbtafel VI. 
Rolle, als Gegenstand der Darstellung und als Repertoire von Methoden zur Darstellung. ${ }^{21}$

Skupins Karte wird in einem komplexen technischen Prozess berechnet, der Schritt für Schritt abläuft, jedoch in einem komplexen Verhältnis zur Form der Karte steht. Das algorithmische Verfahren kann an dieser Stelle nur in seinen wesentlichen Schritten und sehr vereinfacht skizziert werden. Jede Visualisierung von Texten muss in einem ersten Schritt die Texte in numerische Daten verwandeln, die als Grundlage für die weiteren Berechnungen dienen. Skupin benutzt eine bibliometrische Methode zur Auswertung von Texten: Die Menge der von den Autoren angegebenen Schlagworte wurde zur Grundlage für eine Indexierung genommen. ${ }^{22}$ Abstrakt gesehen werden die Texte dadurch entsprechend der in ihnen vorfindlichen Schlagworte als Punkte in dem hochdimensionalen Raum lokalisiert, der durch die Schlagworte aufgespannt wird und genauso viele Dimensionen hat, wie es Schlagworte gibt. Auch wenn Texte mit einem ähnlichen Vorkommen an Schlagwörtern dabei relativ nahe zueinander zu liegen kommen, hat diese räumliche Repräsentation der Texte mit einer Karte indes noch kaum etwas gemein.

Um ein Kartenbild darzustellen, muss die hochdimensionale Punktemenge in einem nächsten Schritt auf die zwei Dimensionen des Papiers oder eines Displays gebracht werden, wobei die Lage der Punkte zueinander möglichst weitgehend erhalten bleiben sollte. Da die Punktemenge der Abstracts aber eine hohe Dimension hat und zudem irregulärer im Raum verteilt sein dürfte als Punkte auf einer Kugeloberfläche, kommen geometrische Projektionen dafür nicht in Frage. Skupin setzt stattdessen einen sehr allgemeinen und komplexen Algorithmus ein, der von Teuvo Kohonen entwickelt wurde. ${ }^{23}$ Dieser Algorithmus soll in der Künstlichen Intelligenz einen unüberwachten Lernprozess simulieren, indem ein zweidimensionales, so genanntes neuronales Netz daraufhin trainiert wird, eine möglichst getreue Repräsentation der hochdimensionalen Ausgangsdaten darzustellen, ohne dass ein Ergebnis im vorhinein gegeben wäre und in Form eines Feedbacks in den Prozess eingespeist würde. Das wiederholte Training des Netzes konver-

21 Siehe dazu auch den programmatischen Artikel André Skupin: From Metaphor to Method: Cartographic Perspectives on Information Visualization. In: S. F. Roth, D. A. Keim (Hg.): Proceedings IEEE Symposium on Information Visualization (InfoVis2000), 9-10 October, 2000, Salt Lake City, Utah, Los Alamitos et al., 2000, S. 91-97. Siehe zudem zur Übersicht möglicher interdisziplinärer Schnittpunkte André Skupin und Sara Irina Fabrikant: Spatialization Methods: A Cartographic Research Agenda for Non-geographic Information Visualization. In: Cartography and Geographic Information Science 30 (2003), S. 95-115.

22 Siehe für die Details dieser Indexierung Skupin 2002 (wie Anm. 18), S. 51.

23 Siehe Teuvo Kohonen: Self-Organizing Maps, Berlin, Heidelberg 1995, insbesondere S. 77130 sowie einleitend André Skupin und Pragya Agarwal: Introduction: What is a Self-Organizing Map?. In: dies. (Hg.): Self-Organising Maps: Applications in Information Science, Chichester et al. 2008, S. 1-20; hier S. 2-14. 
giert ohne jeden externen Eingriff und hat eine zweidimensionale Repräsentation der Ausgangsdaten zum Ergebnis, da jeder Text schließlich einem so genannten Neuron zugeordnet ist.

Ein solches zweidimensionales Resultat von Kohonens Algorithmus scheint einer Karte näher als die Ausgangsdaten und wird meist auch als mehr oder minder abstrakte Karte dargestellt. Dennoch sollte nicht vernachlässigt werden, dass eine solche, auch so genannte Kohonen map wenig mit einer Karte im konventionellen Sinne zu tun hat. Geografische Karten entstehen durch eine geometrische Projektion, durch die die Kugeloberfläche eins zu eins auf die ebene Fläche abgebildet wird. Die grafischen Konstellationen im Bild sind daher recht eng verknüpft mit den Relationen im geografischen Datenmaterial und den Verhältnissen auf der Erdoberfläche. ${ }^{24}$ Dagegen wird durch den Algorithmus Kohonens zunächst jeder Text einem Neuron zugeordnet, dem eine umgrenzte Fläche, aber kein Punkt entspricht. Die Lokalisierung bleibt somit unscharf, weshalb sich Skupin damit behilft, dem Text innerhalb der Fläche eine zufällige Position zuzuweisen. ${ }^{25}$ Ein wesentlicher Unterschied besteht darin, dass kartografische Projektionen Kugelfläche und Kartenbild in einem Schritt aufeinander abbilden, während sich die zweidimensionale Kohonen map durch die Konvergenz eines iterativen Prozesses ergibt und deshalb terminologisch präziser auch selforganizing map genannt wird. In der Konsequenz ist das Verhältnis zwischen den darstellenden Konstellationen im Bild und den dargestellten Bezügen schwerer zu fassen. Skupins Visualisierung sieht eher wie eine Karte aus, als dass sie wie eine Karte entstünde. Die Bezeichnung 'Karter begründet sich daher im Hinblick auf die Konvention der Darstellung und nicht in deren Herstellungsweise. Sie stellt eine der visual metaphors dar, deren sich Visualisierungen von Informationen des Öfteren bedienen, um abstrakte Daten unterschiedlichsten Charakters informativ ins Bild zu setzen. ${ }^{26}$

Wie Skupin in jüngeren Arbeiten zeigt, stehen die Eigenschaften von Kohonens Algorithmus jedoch in einer spezifischen Spannung zur konventionellen Darstellung von Karten. Die self organizing map einer hochdimensionalen Punktemenge erhält in der zweidimensionalen Repräsentation nicht - wie andere Algorithmen - in erster Linie die Distanzen zwischen den Punkten. Vielmehr gibt sie eher die topologische Struktur wieder und nutzt dazu die vorgegebene Fläche nahezu gänzlich, was erhebliche Verzerrungen der räumlichen Distanzen zur Folge

24 Siehe Skupin, Fabrikant 2003 (wie Anm. 21), S. 97-101.

25 Siehe Skupin 2002 (wie Anm. 18), S. 53.

26 Siehe für einen Überblick zur 'map metaphor` zum Beispiel Skupin 2000 (wie Anm. 21), S. 91. Zahlreiche, eher einfache Beispiele finden sich bei Edward R. Tufte: The Visual Display of Quantitative Information, Cheshire 22001 , S. 16-26 sowie bei Jacques Bertin: Graphische Semiologie. Diagramme Netze Karten, Berlin, New York 1974, S. 364-385. 
hat. ${ }^{27}$ Diese Eigenschaft steht aber in Kontrast zur gewohnten Darstellung von Karten. Sobald Punkte in einer Karte lokalisiert werden, sind deren Beziehungen gerade durch die Distanzen im flächigen Kartenbild augenfällig gegeben: Lokalisierte Punkte sind notwendigerweise einander nah oder fern und stehen schon durch ihre Entfernung miteinander in Beziehung. Skupin zitiert selbst das "first law of geography", das der Geograf und Kartograf Waldo R. Tobler in einem Text von 1970 wie folgt formuliert hat: »everything is related to everything else, but near things are more related than distant things. ${ }^{28}$ Durch die Darstellung der Punkte in der Form einer Karte entstehen folglich nicht nur Beziehungen, die weder durch die Punktemenge noch in den Texten oder ihrer Verschlagwortung ohne weiteres gegeben sind. Die Karte bringt auf der grafischen Ebene zudem Beziehungen hervor, die wegen der Eigenschaften einer self organizing map kaum als Verhältnisse im Dargestellten betrachtet werden können. Doch die svisuelle Metapher ' der Karte legt das Gegenteil nah. Die Kartendarstellung wird nämlich nicht zuletzt deshalb benutzt, weil wir gewohnt sind, mit Karten umzugehen, ${ }^{29}$ so dass es ebenso gewollt wie unvermeidlich scheint, dass wir im Kartenbild trotz allem dargestellte Distanzen erblicken. Sie erweist sich damit als entscheidend für die konkrete Form des eröffneten visuellen Bezugs auf die dargestellten Verhältnisse. Die grafische Darstellung und die algorithmische Berechnung arbeiten in dieser Karte der Geografie somit einander entgegen, mag dieser Widerstreit zwischen Darstellung und Herstellung zunächst auch nicht sichtbar werden.

Die Form der Karte motiviert darüber hinaus weitere Elemente von Skupins Visualisierung. In Anlehnung an die politische Ordnung der Geografie in Form von Ländern und Regionen werden auf der Grundlage der bisherigen Ergebnisse Gruppen beziehungsweise so genannte cluster von Texten berechnet, die ein ähnliches Vorkommen von Schlagwörtern aufweisen (siehe Abb. 4). ${ }^{30}$ Skupin diskutiert die Ergebnisse unterschiedlicher Algorithmen und Parametrisierungen, um letztlich in der Karte Grenzen zu ziehen und damit weitere Beziehungen in das Bild einzuschreiben. Mit dieser Grenzziehung verlieren die Relationen in der Fläche ihre Homogenität. Der kontinuierlichen Distanz zwischen den Texten wird eine zweite, diskrete Ordnung der Zugehörigkeit zu einem begrenzten Territorium überlagert.

27 Ausgehend vom Problem der kartografischen Projektion zeigt dies Skupin, indem er eine Weltkarte durch den Kohonen Algorithmus in eine self-organizing map transformiert, die zuallererst die - erheblichen - Verzerrungen durch den Algorithmus darstellt. Siehe André Skupin: A Novel Map Projection Using an Artificial Neutral Network. In: Proceedings of 21st International Cartographic Conference, Durban, South Africa, August 10-16, 2003, S. 1165-1172 (verfügbar auf der Homepage des Autors).

28 Siehe Skupin 2000 (wie Anm. 21), S. 92 und W. R. Tobler: A Computer Movie Simulating Urban Growth in the Detroit Region. In: Economic Geography 46 (1970), Supplement, S. 234-240; hier S. 236.

29 Siehe Skupin 2002 (wie Anm. 18), S. 50 und 58.

30 Siehe ebd., S. 53-55. 


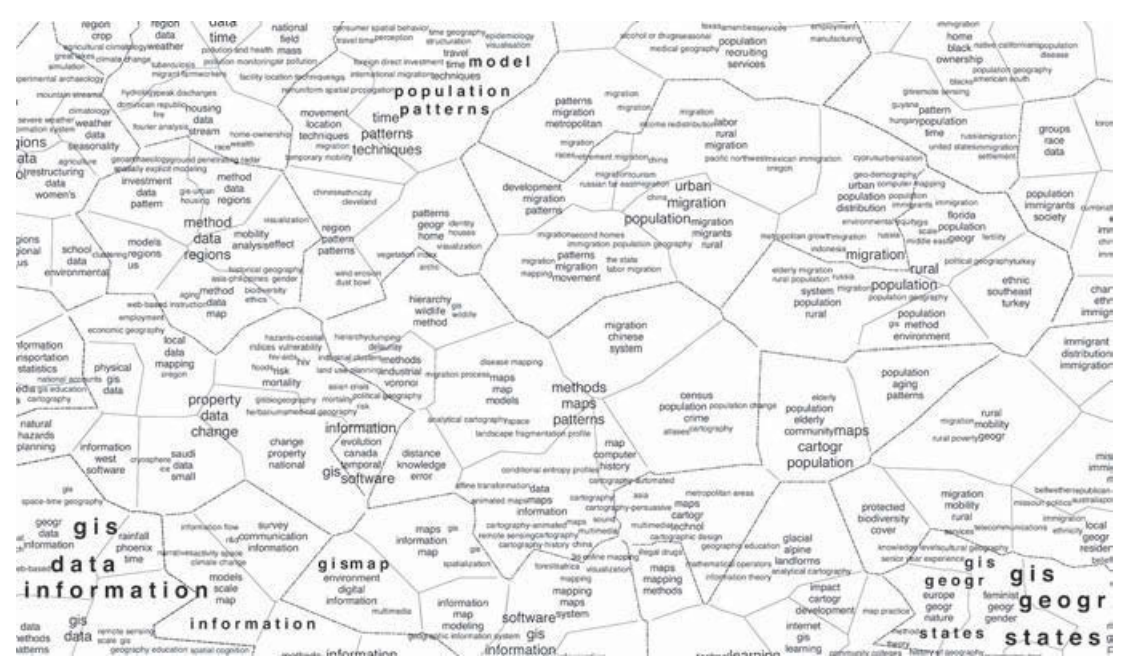

Abb. 4: Detail aus Abbildung 3.

Da das clustering hierarchisch ist und die cluster bis in eine bestimmte Tiefe nochmals unterteilt sind, wird zugleich eine Art diskreter Distanz eingeführt: Um diese Distanz zwischen zwei Texten zu bestimmen, geht man vom ersten Text zum nächsten größeren cluster hinauf, der beide Texte enthält, und steigt bis zur niedrigsten Ebene zum zweiten Text wieder hinab. Die ganzzahlige Länge des Wegs entspricht der baumartigen Struktur der ineinander geschachtelten Gruppen und Untergruppen sowie der inhärenten Unterteilung des ebenen Kartenbilds. Sie kann als diskrete Bestimmung von Distanz verstanden werden und überlagert sich der kontinuierlichen Ferne oder Nähe in der Fläche.

Diese Überlagerung ist in der Visualisierung von Texten anders beschaffen als bei der üblichen geografischen Karte mit ihren Nationalstaaten. In diesem Fall wird der Geografie im Grunde nämlich eine zweite politische Ordnung schlicht überblendet, ohne irgendwelche Interferenzen oder Konflikte zur Folge zu haben: Dass ein Dorf einen genauen Ort und geografische Beziehungen zu anderen Dörfern hat, wird in keiner Weise davon tangiert, welchen politischen Körperschaften die Ortschaften angehören. Auf ähnlich unproblematische Weise können vielerlei Daten in thematischen Karten dargestellt werden, sofern diese Daten auf einen geografischen Ort bezogen werden können. Im Falle von Skupins Visualisierung ist die Sachlage komplizierter, weil hier nicht einfach eine zweite Ordnung ins Spiel kommt. Vielmehr ist es ein und derselbe Raum von Texten, der zweimal gegliedert wird, so dass unter Umständen verschiedene, miteinander konfligierende Beziehungen entstehen. Zwei Texte können beispielsweise nahe beieinander liegen, aber zugleich unterschiedlichen clustern angehören. Ist einer der beiden Texte nun enger verwandt mit einem anderen Text, der zwar in größerer Entfernung zu liegen kam, aber demselben cluster angehört? Die Überlagerung zweier 
Ordnungen in einem Raum schafft hier Interferenzen und Zweideutigkeiten, die letztlich die Frage zuspitzen, in welcher Weise die möglicherweise interferierenden Beziehungen im Bild überhaupt auf die darzustellenden Beziehungen zwischen den Texten bezogen werden können.

Ein genauerer Blick auf Skupins Karte zeigt folglich, dass der zunächst vertraut wirkende Umgang mit der Bildfläche innere Konflikte zeitigt, die im Hinblick auf die Funktion der Darstellung in zweierlei, miteinander verschränkten Dimensionen als maßlos begriffen werden können: Wegen der iterativen Berechnung der Lokalisierung der Punkte, aber auch aufgrund der vielen Möglichkeiten eines clustering ist zum einen der Übergang von den Daten zum Bild und den darstellenden Konstellationen fraglich; zum anderen ist aber auch im Hinblick auf die im Bild entstehenden und unter Umständen konfligierenden Beziehungen unklar, wie überhaupt der im Visuellen eröffnete Bezug auf das Dargestellte eindeutig zu bestimmen sein soll. Im Übergang von den Daten zur Darstellung, aber auch ausgehend von der Darstellung und mit Hinblick auf das Dargestellte scheint das Bild insofern maßlos, als die Übergänge ungesichert sind und kein Maß etwaige Abweichungen abzuschätzen hilft. Diese Maßlosigkeit beruht nicht allein in der Gestalt der Visualisierung, die durchaus nicht maßlos ist, sieht man einmal davon ab, dass sie wie jede Karte in der Übersicht überladen wirkt. Die Maßlosigkeit hat ihren Ort im Scharnier zwischen Herstellung und Gestaltung, zwischen Sichtbarmachung und Sichtbarkeit des Bildes.

Die Komplexität von Skupins Visualisierung zeigt sich noch deutlicher, wenn man einen weiteren Schritt ihrer Entstehung verfolgt. Aus den charakteristischen Wortprofilen der Texte wurden Schlagworte berechnet, um die cluster und ihre Untergliederungen zu beschriften. Während geografische Karten Länder benennen und damit die territorialen Einteilungen nochmals bestätigen, erweist sich die Beschriftung von Skupins Karte der Welt der Geografie als recht zwiespältig, weil die errechneten Schlagwörter einander widerstrebenden Anforderungen genügen müssen: Sie sollten zugleich hinreichend unscharf sein, um innere Gemeinsamkeiten zu erhalten, und genügend scharf, um Differenzen zu den benachbarten Gebieten zu markieren. ${ }^{31}$ Eine genauere Betrachtung der Beschriftungen führt diese Schwierigkeit vor Augen: Es fällt sofort auf, dass die Bezeichnungen wiederholt, ja häufig vorkommen, auf den verschiedenen Ebenen der Hierarchie ebenso wie auf unterschiedlichen Seiten der Grenzen (siehe Abb. 5). Skupin selbst weist auf diese auffälligen Wiederholungen hin, die er durch die bereits angerissene Überlagerung der kontinuierlichen self organizing map und der diskreten Teilung des hierarchischen clusterings erklärt. ${ }^{32}$ Die angewandten Algorithmen geraten in

31 Dieser Grenzgang schlägt sich in einer Formel mit heuristischen Gewichtungen nieder, die letztlich rein pragmatischen Charakters ist. Siehe Skupin 2002 (wie Anm. 18), S. 55.

$32 "$ For example, notice the appearance of similar labels near the peripheries of neighboring clusters [...], indicative of the tension between a strict partitioning mechanism and the continuous nature of the self-organizing map.« Skupin 2004 (wie Anm. 18), S. 5276. 


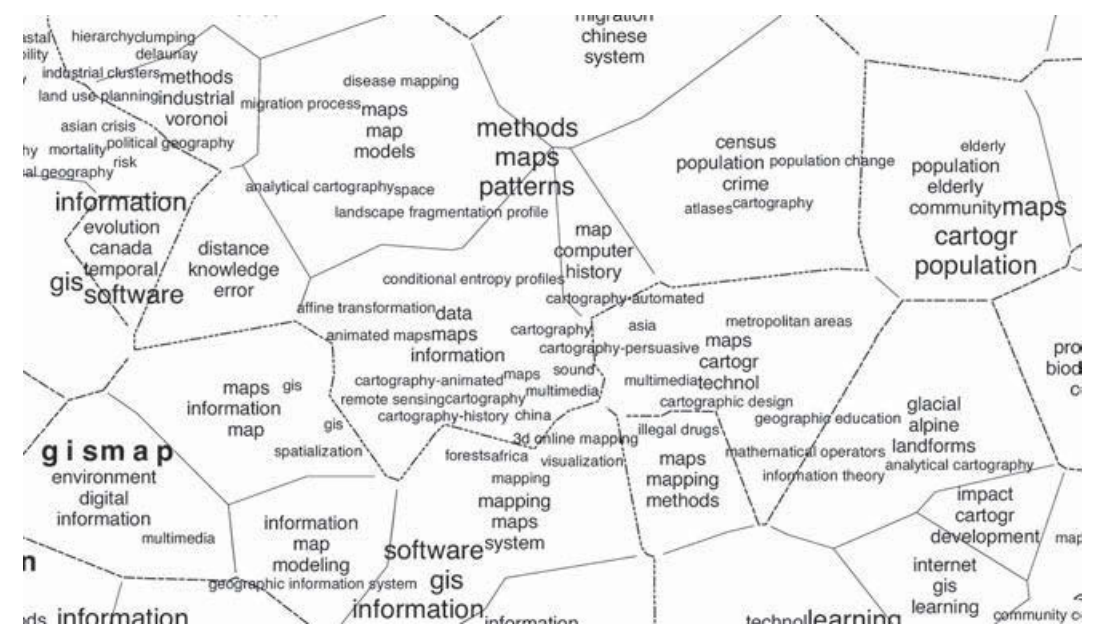

Abb. 5: Detail aus Abbildung 3.

Konflikt miteinander - und in diesem Fall kommt der Konflikt im Bild zum Ausdruck. Dadurch gibt die Karte aber einen sichtbaren Anlass dazu, die grafische Suggestion der räumlichen Trennung und eindeutigen Zuordnung von Texten in Frage zu stellen, also eine Annahme zu bezweifeln, die für eine Visualisierung von Texten, Themen und Begriffen alles andere als selbstverständlich scheint. Die rvisuelle Metapher der Karte gerät in Konflikt mit der Herstellung, so dass der visuelle Bezug der Darstellung sichtbar fragwürdig wird.

Skupin versucht in jüngeren Arbeiten dieses Problem der inkohärenten Beschriftungen wiederum mit kartografischen Mitteln in den Griff zu bekommen. Er lässt zusätzlich die interne thematische Kohärenz der cluster berechnen und visualisiert sie als topografische Struktur: Hohe Kohärenzen bilden Berge, mangelnde Kohärenzen dagegen Täler und Seen. ${ }^{33}$ Auf diese Weise stellt die Karte nun auch die lokale Inkohärenz oder Kohärenz der Regionen dar. Durch diese figurative Darstellung sollen die der Visualisierung inhärenten Spannungen und Konflikte eingeholt werden. Sie sind indes in der Herstellung und Darstellung dieser Sichtbarmachung angelegt und schreiben sich in das Bild sichtbar ein. Sie durchziehen den Bildraum und lassen ihn problematisch erscheinen. In der Konsequenz konfligieren die grafischen Beziehungen im Bild, und ihre sichtbare Ordnung widerstreitet sich selbst, so dass der visuelle Bezug auf die dargestellten Verhältnisse ungewiss ist. Indem Inkohärenzen auf Orte im Raum beschränkt und figurativ dargestellt werden, wird die Maßlosigkeit dieser Visualisierung, die auf den ersten Blick so gar nicht ins Auge fallen mag, nur scheinbar gebändigt.

33 Siehe ebd., S. 5277 sowie Skupin, Fabrikant 2003 (wie Anm. 21), S. 110-111. 


\section{Folgerungen}

Die Annahme, dass Bilder, die etwas darstellen sollen, kaum maßlos sein können, dürfte weit verbreitet sein. Und tatsächlich stößt insbesondere bei wissenschaftlichen Visualisierungen der erste Blick kaum auf etwas Maßloses. Diese Auffassung setzt allerdings voraus, dass das Bild schlicht dasjenige ist, was ich vor mir sehe. Es scheint, als wäre dem wenig entgegenzusetzen. Jedoch spielt die Entstehung des Bildes in den Sinn und die Wahrnehmung des Bildes hinein, so dass der Ort des Bildes und seiner Maßlosigkeit komplexer gefasst werden muss. Insbesondere bei darstellenden Bildern sind die Mittel der Sichtbarmachung zentral, weil sie allein den Bezug auf das Dargestellte absichern könnten. Sie stoßen in der Darstellung allerdings auf ein kritisches Moment, da Bezüge verloren gehen können und je nach grafischer Form zugleich neue Beziehungen geschaffen werden. Ausgehend vom resultierenden Bild ist der Bezug auf den dargestellten Gegenstand deshalb insofern ein visueller Bezug, als er stets von den Konstellationen im Bild ausgeht, also sowohl in der Herstellung als auch in der Darstellung, in der Sichtbarmachung wie in der Sichtbarkeit des Bildes gründet. Eine Charakterisierung der Sichtbarmachung, die die Sichtbarkeit des Bildes einschließt, kann daher die Darstellung im Hinblick auf das Dargestellte durchaus als maßlos begreifen. ${ }^{34}$ Diese Maßlosigkeit hat ihren Ort aber nicht allein im sichtbaren Bild, sondern auch in dessen Herstellung.

Anhand von Skupins Visualisierung hat sich darüber hinaus eine spezifischere und komplexere Maßlosigkeit gezeigt. In dieser Karte überlagern sich verschiedene Ordnungen des Raumes und seiner inhärenten Beziehungen. Es ergeben sich sichtbar Konflikte, die das Verhältnis der darstellenden grafischen Konstellationen zu den darzustellenden Strukturen fragwürdig werden lassen und damit den visuellen Bezug der Darstellung auf das Dargestellte im Sichtbaren selbst aushöhlen. Ähnliche Phänomene sind der Kunstwissenschaft aus der Geschichte der Perspektivkonstruktion bekannt. Auch die Zentralperspektive charakterisiert sich durch ein Verhältnis von dargestellten und darstellenden Relationen, das insofern maßlos zu nennen ist, als es keinen gesicherten wechselseitigen Übergang gibt. Sie soll zwar zum einen Relationen in einer dreidimensionalen Welt derart ins Bild setzen, dass der Betrachter den Eindruck hat, in einen Raum zu schauen, der sich in Kontinuität zur visuellen Wahrnehmung seiner Umwelt befände. Zugleich kann die Perspektive jedoch wie die kartografische Projektion prinzipiell nicht alle Relationen im Raum bewahren, weshalb Positionen von Gegenständen oder Personen unter Umständen nicht mehr genau zu bestimmen sind. Was in dieser Formulierung einen Verlust an Information anklingen lässt oder im Falle der Karte eine

34 In einer solchen Maßlosigkeit wäre ein zentrales methodisches Problem der Informationsvisualisierung zu sehen, da die Entsprechung der im Bild sichtbaren Strukturen zu den sichtbar zu machenden Strukturen der Daten nicht ihrerseits gemessen und damit jedenfalls im technischen Sinne nicht gewährleistet werden kann. 
mögliche Täuschung mit sich bringen mag, birgt durchaus ein eigenes bildliches und darstellerisches Potential, wie die kunstgeschichtliche Diskussion der Perspektive gezeigt hat. Die Zentralprojektion lässt zum anderen nämlich Beziehungen im flächigen Bild entstehen, die sich für Momente von der Darstellung lösen und selbst in den Vordergrund treten können. Dadurch gerät die darstellende Funktion des Bildes in unauflöslichen Widerstreit mit dessen eigener Sichtbarkeit. Dieser reflexive Grundzug des Bildes kann zum Gegenstand der künstlerischen Gestaltung werden. Wie sich exemplarisch an Masaccios Trinitätsfresko zeigen lässt, kann durch die geschickte Wahl eines Standpunkts und entsprechende Unklarheiten über die Platzierungen von Personen und Gegenständen etwas `dargestellt « werden, das seinen Ort nicht im dreidimensionalen Raum hat. ${ }^{35}$ Die Kunst befolgt in einer solchen Komposition die Perspektivkonstruktion und wendet sie zugleich gegen sich selbst, um zeigen zu können, was kein gemeinsames Maß hat mit dem dargestellten Raum und dem Raum des Betrachters, die scheinbar kontinuierlich ineinander übergehen. Der visuelle Bezug des Bildes, der zunächst durch die ebenso kunstvolle wie strenge Wendung der Perspektive auf ein Unbestimmtes hin geöffnet wurde, wird dadurch im selben Moment auf Transzendentes umgelenkt und in eine höhere Darstellung überführt.

Was künstlerisch genutzt wird, kann sich aber auch unwillkürlich einstellen. Skupins Karte der Geografie führt vor Augen, wie sich in der Überlagerung verschiedener Ordnungen der Bildfläche kaum auflösbare Konflikte zwischen Sichtbarmachung und Sichtbarkeit zeigen. Ungewollt lässt sie deutlich werden, wie die im Bild sichtbaren darstellenden Konstellationen in Widerstreit geraten zur Darstellung derjenigen Relationen, die sichtbar gemacht werden sollten. In diesem Moment hat die Darstellung kein Maß mehr gemein mit dem Dargestellten, das den visuellen Bezug zum Gegenstand kontrollieren könnte. Diese Maßlosigkeit hat ihren Ort folglich zwischen der Sichtbarmachung und der Sichtbarkeit des Bildes. Anders gesagt verdankt sie sich dem doppelten Sinn der Darstellung von Bildern, die nur etwas darstellen, wenn sie selbst hergestellt oder dargestellt werden. ${ }^{36}$ Diese beiden Seiten des Darstellens sind in einem komplexen Prozess untrennbar aufeinander bezogen und interferieren unvermeidbar miteinander, wobei sich der resultierende maßlose Charakter der Darstellung im Bild nicht aufdrängen muss, aber doch zeigen kann. Eine Ästhetik, die diese Maßlosigkeit einfangen wollte, dürfte sich deshalb nicht mit dem sichtbaren Bild begnügen. Sie müsste auch des-

35 Siehe stellvertretend Klaus Krüger: Das Bild als Schleier des Unsichtbaren. Ästhetische Illusion in der Kunst der frühen Neuzeit in Italien, München 2001, S. 34-37 sowie zur »Inkommensurabilität« des Dargestellten Johannes Grave: Brunelleschi’s Perspective Panels. Rupture and Continuity in the History of the Image. In: Harry Schnitker, Pierre Péporte, Alexander C. Lee (Hg.): Renaissance? Perceptions of Continuity and Discontinuity in Europe, Leiden 2009 (im Druck).

36 Siehe dazu auch Hans-Jörg Rheinberger: Experimentalsysteme und epistemische Dinge. Eine Geschichte der Proteinsynthese im Reagenzglas, Frankfurt am Main 2006, S. 127-128. 
sen Herstellungsweise einbeziehen, ohne allerdings den Begriff des Ästhetischen darauf zu reduzieren, dass Bilder hergestellt und daher auch gestaltet sind. ${ }^{37}$ Vielmehr muss eine Ästhetik am bildlichen Erscheinen festhalten, dabei allerdings die Herstellung des Bildes und im Falle von Darstellungen die Prozesse der Sichtbarmachung einbeziehen. Eine Ästhetik des Bildes wird es deshalb nicht nur mit den Bedingungen unserer Wahrnehmung, sondern auch mit den Bedingungen des Bildlichen und ihren spezifischen Unterschieden zu tun haben.

37 Nach dieser eher oberflächlichen Auffassung hätte das Bild eine ästhetische Dimension, weil die Herstellung ästhetische Entscheidungen wie die Farbwahl und ähnliches impliziert, seien sie nun bewusst getroffen oder durch technische Gegebenheiten nahe gelegt oder schon gefallen. Ausgehend von der Frage nach der Maßlosigkeit wird deutlich, dass der Begriff des Ästhetischen auch keineswegs hinauslaufen muss auf "the very fabric of realism: the work of composing visible coherences, discriminating differences, consolidating entities, and establishing evident relations«, wie es einst Lynch und Edgerton 1988 (wie Anm. 6), S. 212 vorgeschlagen haben. 